


\title{
On the Ziglin-Yoshida Analysis for Some Classes of Homogeneous Hamiltonian Systems
}

\author{
M. A. Almeida, I. C. Moreira* and F. C. Santos \\ Caixa Postal 68528, Instituto de Física, UFRJ \\ 21945-170, Rio de Janeiro, Brazil
}

Received 5 August, 1997

In this paper we use the Ziglin-Yoshida method to discuss the determination of nonintegrability domains for some classes of homogeneous hamiltonian systems. In particular, we demonstrate the non-integrability of the Störmer problem through the reduction of the system to a two-dimensional homogeneous potential. We have also found the nonintegrability domains of potentials of the form $V=q_{1}^{m} q_{2}^{n}\left(A q_{1}^{2}+B q_{2}^{2}\right)^{p}$ and $V=q_{1}^{m} q_{2}^{n} q_{3}^{p}$.

\section{Introduction}

In the last two decades or so, there has been an increasing interest on the analysis of the integrability of dynamical systems. An integrable system is characterized by regular (periodic) behavior for (almost) all initial conditions and all time; in this case, we can get global information on its long-term behavior. Non-integrable systems have regions in the phase space where the motion is irregular, or chaotic; in the context of dynamical systems, chaos means an extreme sensitivity of the solutions to the choice of initial conditions. Therefore, it is an important problem to find methods for the identification of integrable or non-integrable systems.

The notion of integrability is related to the existence of first integrals. So the question above can be stated in the following form: how can we identify the values of the parameters for which the equations of motion have (or not) first integrals? Several methods for the identification of classes of integrable systems have been developed: singularity analysis [1], direct construction of polynomial first integrals $[2,3]$, linear compatibility method [4], Lie and Noether symmetry analysis $[5,6]$, construction of Lax's pairs [7], the Carlemann embedding method [8], the quasimonomial formalism [9] etc. On the other side, the proof of the non-integrability of a given system is usually a difficult task. The tentative of finding a generic algorithm for proving the non-integrability of a general dynamical system has not yet been accomplished and there are some evidences that, in the general case, the mathematical problem belongs to the class of undecidable mathematical problems. However, particular methods have been developed for analyzing the non-integrability of hamiltonian systems.

Hamiltonian systems have a particular importance in physics and possess a peculiar mathematical property: the sympletic structure. A hamiltonian system with $\mathrm{N}$ degrees of freedom is Liouville integrable whenever $\mathrm{N}$ global first integrals, in involution, can be obtained. Furthermore, if the level set (intersection) of these integrals is compact and their gradient vectors are linearly independent on the level set for a given initial condition, then the motion is expressed as a quasiperiodic motion on an N-dimensional torus [10]. The proof of the integrability of a hamiltonian system is based on the exhibition of these $\mathrm{N}$ integrals of motion.

An important procedure in this direction was established by Melnikov, starting from Poincaré's results; it permits to detect the non-integrability of a perturbed hamiltonian system. Poincaré introduced the concepts of homoclinic and heteroclinic orbits, linking fixed points, and showed that perturbations of these orbits are sources of complex behaviors in dynamical systems. The importance of homoclinic orbits and of the transversal homoclinic interception - i.e, the transversal interception of unstable and stable manifolds of a fixed

\footnotetext{
*E-mail: ildeu@if.ufrj.br
} 
point - was definitively established with the SmaleBirkhoff theorem. This theorem shows that the existence of transversal homoclinic interceptions is a sufficient condition for the existence of Cantor invariant sets in which the periodic orbits are dense.

Melnikov's method gives a criterion for the existence of a transversal homoclinic interception in the Poincaré's section defined by the flux; a function which measures the separation between the unstable and stable manifolds allows the detection of the interception. A basic supposition for the application of this method is that the non-perturbed Hamiltonian must have a known separatrix, a situation that is not always satisfied. This method is well-known and is exposed in some recent textbooks on non-linear systems [11]. Another possibility for proving the non-integrability of a dynamical system is to prove directly the existence, in the phase space, of a structure that is equivalent to a Bernoulli shift or to a Smale horse-shoe.

Here we will limit ourselves to discuss another method for the detection of non-integrable hamiltonian systems, the so-called Ziglin-Yoshida analysis, and to make some applications of this method for specific classes of homogeneous Hamiltonians with two and three degrees of freedom. Some years ago, Ziglin [12] has proven a powerful theorem which gives a necessary condition for integrability and can thus be used to prove the non-integrability of a given hamiltonian system. He considered the Hamiltonian as a complex function, so that the solutions can be taken as analytical functions of the time $t$. He analyzed the monodromy properties around particular solutions of the system and the conditions for the non-existence of an additional integral of motion. In Ziglin's original paper the motion of a rigid body around a fixed point was considered as an example.

Significant advances in Ziglin's approach were made by Yoshida [13]: he initially proved a theorem concerning the non-integrability of homogeneous hamiltonian systems, with two degrees of freedom, where the conditions coming from Ziglin's results can be put in an explicit and operational form. Some time latter, he established a sufficient condition for the non-existence of an additional first integral, global and analytical, for homogeneous Hamiltonians with $\mathrm{N}$ degrees of freedom [14]. In the last years the non-integrability domains of several hamiltonian systems were discussed as applications of Ziglin-Yoshida's approach: Hénon-Heiles system [15]; some generalized Toda lattices [16]; the one- dimensional three body problem [13]; some perturbed Kepler potentials [17]; the swinging Atwood machine $[18,19]$; non-homogeneous polynomial potentials of degree 3 or 4 [20]; truncated Toda lattice of any order [20]; Störmer problem [21]; two-dimensional homogeneous Hamiltonians with a velocity dependent potential [22]; the motion of a satellite around a planet with an arbitrary shape [23]. We note that, in some of these cases, the initial hamiltonian system has been transformed to a homogeneous one by using a coordinate transformation.

\section{Ziglin-Yoshida analysis for homogeneous Hamiltonians}

The theorem proved by Ziglin depends on non-local analysis and leads to necessary conditions for an analytical Hamiltonian to have global analytical first integrals in the coordinates $\left(p_{i}, q_{i}\right)$. The basic idea is to get integrability conditions for the variational equations, around a particular solution, showing that, if they are not satisfied, a hamiltonian system with $\mathrm{N}$ degrees of freedom cannot have a complete set of $\mathrm{N}$ global analytical first integrals (including the Hamiltonian itself). The variational equations are obtained by linearization of the harniltonian equations of motion, around a particular solution, i.e, by making a very small variation in the initial conditions and studying the evolution of the separation of neighboring trajectories. If this evolution is sufficiently complicated, as measured by the behavior of the monodromy matrix, it is possible to demonstrate the non-existence of additional analytical firsts integrals.

The fact that Ziglin's method is based on the behavior of the variational equations, which are linear equations, is one of its advantages because we must consider only first order expansions. However, one important limitation of this procedure comes from this same fact: it does not allow the analysis of situations were the integrability property is broken at higher orders. Some interesting links between Ziglin's results and the singularity analysis, through Kowalewskaia-Painlevé test, were discussed in reference [24]. We will not discuss here Ziglin's main theorem. Yoshida [13] furnished a general presentation and a new demonstration of this theorem; the original formulation of the theorem can be find in [12]. We will expose now Yoshida's results concerning the application of Ziglin's theorem for homogeneous Hamiltonians. We begin with homogeneous hamiltonian systems with two degrees of freedom. The hamil- 
tonian equations for a two-dimensional system have the form

$$
\frac{d q_{i}}{d t}=\frac{\partial H}{\partial p_{i}}, \quad \frac{d p_{i}}{d t}=-\frac{\partial H}{\partial q_{i}}
$$

with

$$
H=\left(p_{1}^{2}+p_{2}^{2}\right) / 2+V\left(q_{1}, q_{2}\right) .
$$

We will make the supposition that the potential $V\left(q_{1}, q_{2}\right)$ is a homogeneous potential:

$$
V\left(a q_{i}\right)=a^{k} V\left(q_{i}\right)
$$

There exists, in that case, a particular solution of the equations (1) with the form

$$
q_{i}=c_{i} f(t), \quad p_{i}=c_{i} f^{\prime}(t),
$$

where $f(t)$ is solution of the equation

$$
f^{\prime}+f^{k-1}=0
$$

and the constant vector $c_{i}=\left(c_{1}, c_{2}\right)$ is the solution of the algebraic equation

$$
c_{i}=\left(\partial V / \partial q_{i}\right)\left(c_{1}, c_{2}\right) .
$$

The variational equation of (1), around the particular solution (3), will be

$$
\frac{d \xi_{i}}{d t}=\eta_{i}, \quad \frac{d \eta_{i}}{d t}=-f^{k-2} \mathbf{V}_{i j} \xi_{j} \Rightarrow \frac{d^{2} \xi_{i}}{d t^{2}}=-f^{k-2} \mathbf{V}_{i j} \xi_{j}
$$

where the Hessian matrix $\mathbf{V}_{i j}=\partial^{2} V / \partial q_{i} \partial q_{j}$ is calculated in $q_{i}=c_{i}$, and

$$
\delta q_{i}=\xi_{i}, \quad \delta p_{i}=\eta_{i}
$$

With the diagonalization of $\mathbf{V}_{i j}$ and with the determination of their eigenvectors and eigenvalues, equation (6) can be expressed in terms of two variational equations; the first one is called normal variational equation and the second is the tangential variational equation:

$$
d^{2} \xi_{1} / d t^{2}+\lambda f^{k-2} \xi_{1}=0
$$

$$
d^{2} \xi_{2} / d t^{2}+(k-1) f^{k-2} \xi_{2}=0
$$

where

$$
\lambda=\operatorname{Tr}\left[\mathbf{V}_{i j}\left(c_{1}, c_{2}\right)\right]-(k-1) .
$$

By applying Ziglin's results to the case of two-dimensional homogeneous hamiltonian systems, Yoshida deduced some relations between the values of the integrability coefficient $\lambda$ and the existence of domains of the parameters for which the system has not a second analytical first integral, besides the energy. The following theorem, whose demonstration can be find in reference [13], summarizes Yoshida's results:

\section{Theorem I}

Let $V\left(q_{1}, q_{2}\right)$ be a homogeneous potential function of an integer degree $k$ and compute the quantity (integrability coefficient) $\lambda$ defined by

$$
\lambda=\operatorname{Tr}\left[\mathbf{V}_{i j}\left(c_{1}, c_{2}\right)\right]-(k-1),
$$

where $\mathbf{V}_{i j}$ is the Hessian matrix $V\left(q_{1}, q_{2}\right)$ and $\left(c_{1}, c_{2}\right)$ is a solution of the algebraic equations

$$
\begin{aligned}
& c_{1}=\left(\partial V / \partial q_{1}\right)\left(c_{1}, c_{2}\right) \\
& c_{2}=\left(\partial V / \partial q_{2}\right)\left(c_{1}, c_{2}\right)
\end{aligned}
$$

If $\lambda$ is in the region $S_{k}$ defined below, then the two degrees of freedom Hamiltonian system

$$
H=\left(p_{1}^{2}+p_{2}^{2}\right) / 2+V\left(q_{1}, q_{2}\right)
$$

is non-integrable, i.e., there cannot exist an additional integral which is complex analytic in $\left(q_{1}, q_{2}\right)$ and $\left(p_{1}, p_{2}\right)$. The regions $S_{k}$ are defined as follows:

(i) $k^{3} \geq 3$

$S_{k}=\{\lambda<0,1<\lambda<k-1, k+2<\lambda<3 k-2, \ldots, j(j-1) k / 2+j<\lambda<$

$j(j+1) k / 2-j, \ldots\}$;

(ii) $S_{1}=R-\{0,1,3,6, \ldots, j(j+1) / 2, \ldots\}$,

(iii) $S_{-1}=R-\{1,0,-2,-5, \ldots,-j(j+1) / 2+1, \ldots\}$;

(iv) $k \leq-3$

$S_{k}=\{\lambda>1,0>\lambda>|k|+2,|k|-1>\lambda>-3|k|+3, \ldots$,

$-j(j-1)|k| / 2-(j-1)>\lambda>-j(j+1)|k| / 2+(j+1), \ldots\}$; 
where $j$ is an integer.

Note that if $k=0, \pm 2$, such regions are not defined by Theorem I. Indeed when $k=-2$, the system is integrable. In this case, it will admit a second integral of motion with the form:

$$
I=\left(q_{1} p_{2}-q_{2} p_{1}\right)^{2}+2\left(q_{1}^{2}+q_{2}^{2}\right) V\left(q_{1}, q_{2}\right)
$$

This integral can be obtained from the Lie symmetries of the equations of motion which describe the system. They possess the following three symmetry generators:

$$
\begin{aligned}
& U_{1}=\partial / \partial t \\
& U_{2}=2 t \partial / \partial t+q_{1} \partial / \partial q_{1}+q_{2} \partial / \partial q_{2} \\
& U_{3}=t^{2} \partial / \partial t+t q_{1} \partial / \partial q_{1}+t q_{2} \partial / \partial q_{2}
\end{aligned}
$$

These symmetry generators correspond to the time translation symmetry, a scale symmetry and a special conform symmetry, respectively. If $k=0$ or $k=2$, a different analysis is necessary for discussing the integrability or the non-integrability of the system.

\section{Homogeneous Hamiltonians w,m two de-} grees of freedom

We will give now some applications of Theorem I for specific classes of homogeneous hamiltonians systems.

\section{(a) Non-integrability of the Störmer problem}

The analysis of the motion of an electrically charged particle in a dipole magnetic field was considered initially by Störmer [25]. This problem is an important one because of its applications to the case of the Earth's magnetic field [26] (A historical summary of researches on this problem can be found in reference [27]). Several authors searched without success for the three integrals of motion which would give the proof of the integrability of this problem [28]. Dragt and Finn, by using topological and numerical techniques, showed that, in this case, the motion of trapped charged particles is expected to be non-integrable [29]. Recently Jung and Scholz [30] and Jung and Rückerl [31] studied the classical scattering of the same problem and found numerical evidence of chaotic behavior.

Let us consider the non-relativistic motion of a charged particle $q$, with mass $m$, in the field of a magnetic dipole $\mathbf{M}$. The hamiltonian of this system is

$$
H=[\mathbf{p}-(q / c) \mathbf{A}]^{2} / 2 m
$$

with

$$
\begin{aligned}
& \qquad \mathbf{A}=(\mathbf{M} \times \mathbf{r}) / r^{3} \\
& \text { By choosing the } z \text { axis in the direction of } \mathbf{M} \text {, i.e, if } \\
& \mathbf{M}=(0,0, M) \text { we get from }(13) \text { : }
\end{aligned}
$$

$$
H=\left(p_{x}^{2}+p_{y}^{2}+p_{z}^{2}\right) / 2+\left(a / r^{3}\right)\left(y p_{x}-x p_{y}\right)+\left(a^{2} / 2 r^{6}\right)\left(x^{2}+y^{2}\right) .
$$

with $m=1, r=\left(x^{2}+y^{2}+z^{2}\right)^{1 / 2}$ and $a=q M / c$.

The equations of motion of this system are time-independent, axisymmetric and have also a scale symmetry. The following quantities are conserved: the energy and the $z$ component of the angular momentum:

$$
L_{z}=x p_{y}-y p_{x}
$$

If we choose cylindrical coordinates $(\rho, \phi, z)$, the Hamiltonian (14) becomes

$$
H=\left(p_{\rho}^{2}+p_{\phi}^{2} / \rho^{2}+p_{z}^{2}\right) / 2+\left(a^{2} \rho^{2} / 2\right)\left(\rho^{2}+z^{2}\right)^{-3}+a p_{\phi}\left(\rho^{2}+z^{2}\right)^{-3 / 2}
$$


and $p_{\phi}=L_{z}=$ constant.

As far as the motion in $\rho$ and $z$ is concerned we can regard $H\left(p_{\rho}, p_{z}, p_{\phi}=\right.$ constant, $\left.\rho, z\right)$ as a reduced Hamiltonian describing the two-dimensional motion in the $(\rho, z)$ plane. In particular, if we take $p_{\phi}=0$ the reduced Hamiltonian will be

$$
H=\left(p_{\rho}^{2}+p_{z}^{2}\right) / 2+\left(a^{2} \rho^{2} / 2\right)\left(\rho^{2}+z^{2}\right)^{-3}
$$

If the original Hamiltonian (15) is integrable with a third integral of motion, then the reduced Hamiltonian (16) should be integrable too. This reduced Hamiltonian (16) has a homogeneous potential with degree $k=-4$ :

$$
V=\left(a^{2} \rho^{2} / 2\right)\left(\rho^{2}+z^{2}\right)^{-3}
$$

From (8), we get the algebraic equations:

$$
\begin{gathered}
c_{1}=a^{2} c_{1}\left(c_{1}^{2}+c_{2}^{2}\right)^{-3}-3 a^{2} c_{1}^{3}\left(c_{1}^{2}+c_{2}^{2}\right)^{-4} \\
c_{2}=-3 a^{2} c_{1}^{2} c_{2}\left(c_{1}+c_{2}^{2}\right)^{-4}
\end{gathered}
$$

A solution for (18) is

$$
c_{1}=\left(-2 a^{2}\right)^{1 / 6}, \quad c_{2}=0 .
$$

The Hessian matrix becomes

$$
\left|\begin{array}{cc}
-5 & 0 \\
0 & 3 / 2
\end{array}\right|
$$

and, therefore, the integrability coefficient $\lambda$ for the potential (16) will be

$$
\lambda=\operatorname{Tr}\left[\mathbf{V}_{i j}\left(c_{1}, c_{2}\right)\right]-(k-1)=3 / 2 .
$$

In this case, Theorem I gives us the following nonintegrability domains:

$$
S_{-4}=\{\lambda>1,0>\lambda>-2,-5>\lambda>-9, . .\} .
$$

As $\lambda$, from (19), falls in the region $S_{-4}$, we conclude that the potential (17) is a non-integrable one; therefore, the non-integrability of the Störmer problem, described by the Hamiltonian (15), is proved. Another proof of the non-integrability was given independently by Noguera [32], who showed the inclusion of the Benoulli shift as a subsystem of the invariant manifolds of the Lyapounov's orbit in the isolated equilibrium point.

It can be observed that if we make a generalization of the potential (17), by including the possibility of an anisotropy

$$
V=\rho^{2}\left(\rho^{2}+\gamma z^{2}\right)^{-3}
$$

with $\gamma>0$, the integrability coefficient becomes $\gamma=$ $3 \gamma / 2$ and, by Theorem I, the system will be out of the domain of non-integrability if $\gamma \in(0,2 / 3)$, although its integrability is not assured.

(b) Hamiltonians with the form $H=p_{1}^{2} / 2+p_{2}^{2} / 2+$ $q_{1}^{m} q_{2}^{n}\left(A q_{1}^{2}+B q_{2}^{2}\right)^{p}$

We analyze now the non-integrability domains for systems with Hamiltonians with the general form

$$
H=p_{1}^{2} / 2+p_{2}^{2} / 2+q_{1}^{m} q_{2}^{n}\left(A q_{1}^{2}+B q_{2}^{2}\right)^{p}
$$

This kind of Hamiltonian has a homogeneous potential of degree $k=m+n+2 p$. We will suppose that $k$ is an integer. If we take the algebraic equations (8), for this case, we get

$$
\begin{aligned}
& c_{1}=m c_{1}^{m-1} c_{2}^{n}\left(A c_{1}^{2}+B c_{2}^{2}\right)^{p}+2 A p c_{1}^{m+1} c_{2}^{n}\left(A c_{1}^{2}+B c_{2}^{2}\right)^{p-1} \\
& c_{2}=n c_{2}^{m} c_{2}^{n-1}\left(A c_{1}^{2}+B c_{2}^{2}\right)^{p}+2 B p c_{1}^{m} c_{2}^{n+1}\left(A c_{1}^{2}+B c_{2}^{2}\right)^{p-1}
\end{aligned}
$$


As the general situation is very complicated for discussing all possibilities, we will consider some particular cases.

(i) Take $c_{2}=0$ and $c_{1} \neq 0$. This solution occurs if $n=0$. Equations (22) lead to

$$
c_{1}=\left[(m+2 p) A^{p}\right]^{1 /(2-k)} ; \quad c_{2}=0
$$

The potential will have the form

$$
V=q_{1}^{m}\left(A q_{1}^{2}+B q_{2}^{2}\right)^{p}
$$

with degree $k=m+2 p$. If we calculate the integrability coefficient $\lambda$, we find

$$
\lambda=2 B p / k A \text {. }
$$

If $A=B$, we get

$$
\lambda=2 p / k=2 p /(m+2 p) .
$$

The reduced Hamiltonian (16) of the Störmer problem that we have found above is a particular case of (24), with $m=2, p=-3, k=-4, \lambda=3 / 2$. Other interesting particular cases are:

(a) If $k \leq 3(m>0$ and $p>0)$, the integrability coefficient will be within the interval $(0,1)$ and out of the domain of non-integrability furnished by Theorem I;

(b) If $k=m+2 p=1$, then

$$
\lambda=2 p
$$

and the system will be non-integrable if $p \neq$ $3,5,14, \ldots, j(j+1) / 4$;

(c) The potential

$$
V=\left(q_{1}^{2}-q_{2}^{2}\right)^{2}
$$

was considered by Matinyan, Prokhorenko and Sawidy [33], as an particular case coming from time-dependent Yang-Mills equations, with spherical symmetry. It is a non-integrable system as can be seen from the calculation of the integrability coefficient $\lambda=-1$. Theorem I guarantees the non-integrability of this potential that has the degree $k=4$;

(ii) There exists a symmetric case the case (i): $c_{1}=$ $m=0$ and $c_{2} \neq 0$, for the potential

$$
V=q_{2}^{n}\left(A q_{1}^{2}+B q_{2}^{2}\right)^{p}
$$

where

$$
c_{2}=\left[(n+2 p) B^{p}\right]^{1 /(2-k)}
$$

and

$$
\lambda=2 A p / k B
$$

(iii) An important particular case of (21) was analyzed by Yoshida [13]: it occurs if we take $m=n=0$. The potential, in this case, is

$$
V=\left(A q_{1}^{2}+B q_{2}^{2}\right)^{p}
$$

with $k=2 p$.

The integrability coefficient, from (7) and (8), will be

$$
\text { a) } \lambda=B / A, \quad \text { b) } \lambda=A / B \text {. }
$$

In the isotropic case, $A=B$ and $\lambda=1$. The potential, in this case, will be out of the domain of nonintegrability. If $A \neq B, A$ and $B$ being positive, and $k \leq-3$, one of the integrability coefficients will be in the domain of non-integrability. If $k=-1$ we have a confirmation of the non-integrability of the Kepler anisotropic problem. In this case, $A$ and $B$ being arbitrary, the unique possibility of integrability will happen for $A=B$, i.e, for the usual Kepler problem. A similar situation occurs if $k=1$;

(iv) Consider now the Hénon-Heiles homogeneous system:

$$
V=q_{1}^{2} q_{2}+(e / 3) q_{2}^{3}
$$

It is a particular case of (21), with $m=0, n=1$, $A=1, B=e / 3, \quad p=1$. A direct verification shows that equations (22) have the following solutions:
a) $c_{1}=0, \quad c_{2}=1 / e$
b) $c_{1}^{2}=(2-e) / 4, \quad c_{2}=1 / 2$.

They conduce, by Theorem 1 , to the following regions where the system is a non-integrable one:

a) $\{e<0,1<e<2,2 / 7<e<2 / 5, \ldots\}$,

b) $\{e<1,2<e<3,6<e<8, \ldots\}$.

If we put this regions together, we get the nonintegrability domain for potential (32):

$$
\{e<1,1<e<2,2<e<3,6<e<8, \ldots\} \text {. }
$$


Only the cases where $e=1, e=6$ and $e=16$ are known as integrable,

(v) If $m=n$ and $A=B$, the potential, from (21), becomes

$$
V=q_{1}^{n} q_{2}^{n}\left(q_{1}^{2}+q_{2}^{2}\right)^{p}
$$

and the equations (22) have the solution

$$
c_{1}=c_{2}=\left[1 /\left(k 2^{p-1}\right]^{1 /(k-2)}\right.
$$

with $k=2(n+p)$. The integrability coefficient will be

$$
\lambda=(p-n) /(p+n)
$$

In Table 1, we give some particular cases for these conditions.

\section{Table 1}

\begin{tabular}{|c|c|c|}
\hline CONDITIONS & $\begin{array}{c}\text { INTEGRABILITY } \\
\text { COEFFICIENT }\end{array}$ & $\begin{array}{c}\text { POTENTIAL } \\
\mathrm{V}=\mathrm{q}_{1}{ }^{2} \mathrm{q}_{2}{ }^{2}\left(\mathrm{q}_{1}{ }^{2}+\mathrm{q}_{2}{ }^{2}\right)^{\mathrm{p}}\end{array}$ \\
\hline $\mathrm{n}=2, \mathrm{p}=0(\mathrm{k}=4)$ & $\lambda=-1$ & Non-integrable \\
\hline $\mathrm{n}=2, \mathrm{p}=1(\mathrm{k}=6)$ & $\lambda=-1 / 3$ & Non-integrable \\
\hline $\mathrm{n}=2, \mathrm{p} \geq 2(\mathrm{k} \geq 8)$ & $\lambda=(\mathrm{p}-2) /(\mathrm{p}+2) \in[0,1]$ & Indeterminate \\
\hline $\mathrm{n}=2, \mathrm{p} \leq-4(\mathrm{k} \leq-4)$ & $\lambda=(\mathrm{p}-2) /(\mathrm{p}+2)>1$ & Non-integrable \\
\hline
\end{tabular}

We observe that if $V=q_{1}^{n} q_{2}^{n} /\left[q_{1}^{2}+q_{2}^{2}\right]^{(n+1)}$ then $k=-2$ and the system can be integrated.

(vi) If $p=0$, the potential (21) takes the form

$$
V=q_{1}^{m} q_{2}^{n}
$$

and $k=m+n$. We have the following algebraic equations to be satisfied:

$$
c_{1}=m c_{1}^{m-1} c_{2}^{n}, \quad c_{2}=n c_{1}^{m} c_{2}^{n-1}
$$

One solution is

$$
c_{1}=m^{[n-2] /[2(k-2)]} n^{-n[2(k-2)]}, \quad c_{2}=(n / m)^{1 / 2} c_{1}
$$

and the integrability coefficient is $\lambda=-1$.

In Table 2, several particular situations are presented in this case. The integrability (or nonintegrability) was analyzed by using Theorem I; the integrable cases can be found by making the explicit determination of the second integral of motion.

\section{(c) The swinging Atwood machine}

The lagrangian for the so-called swinging Atwood machine (SAM) system, studied by Tufillaro et al. [18], is

$$
L=(1 / 2)(M+m) r^{\prime 2}+(1 / 2) m r^{2} \theta^{\prime 2}-g r[M-m \cos (\theta)] .
$$

The hamiltonian for this system, obtained from (39) and by using the transformations

$$
q_{1}=r \cos (\lambda \theta), \quad q_{2}=r \operatorname{sen}(\lambda \theta)
$$

is

$$
H\left(p_{1}^{2}+p_{2}^{2}\right) / 2+g(m+M)\left(q_{1}^{2}+q_{2}^{2}\right)^{1 / 2}\left\{M-m \cos \left[\lambda^{-1} \operatorname{arctg}\left(q_{1} / q_{2}\right)\right]\right\}
$$


Table 2

\begin{tabular}{|c|c|}
\hline CONDITIONS & POTENTIAL: $V=\mathrm{q}_{1}{ }^{\mathrm{m}} \mathrm{q}_{2}{ }^{\mathrm{n}}$ \\
\hline $\mathrm{n}=\mathrm{m}=1(\mathrm{k}=2)$ & Integrable: $\mathrm{V}=\mathrm{q}_{1} \mathrm{q}_{2}\{$ linear $\}$. Indeterminate by Theorem I \\
\hline $\mathrm{n}=\mathrm{m}=2(\mathrm{k}=4)$ & Non-integrable \\
\hline $\mathrm{n}=\mathrm{m}>2(\mathrm{k}>4)$ & Non-integrable \\
\hline $\mathrm{n}=\mathrm{m} \leq-2$ & Non-integrable \\
\hline $\mathrm{m}+\mathrm{n}=1$ & Non-integrable \\
\hline $\mathrm{m}+\mathrm{n}=0$ & Integrable: $\mathrm{V}=\mathrm{q}_{1} / \mathrm{q}_{2}$. Indeterminate by Theorem I \\
\hline $\mathrm{m}+\mathrm{n}=-1$ & Non-integrable \\
\hline $\mathrm{m}+\mathrm{n}=-2$ & Integrable: $\mathrm{V}=\mathrm{q}_{1}{ }^{\mathrm{m}} \mathrm{q}_{2}{ }^{-(2+m)}$ Indeterminate by Theorem $\mathrm{I}$ \\
\hline $\mathrm{m}+\mathrm{n} \leq-4$ & Non-integrable \\
\hline
\end{tabular}

The potential is a homogeneous one with degree $k=1$.

The resolution of (8), in this case, leads to the following possible values for $c_{1}$ and $c_{2}$ :

$$
\begin{gathered}
c_{1}=[(M-m) /(M+m)]^{1 / 2} \operatorname{sen}(2 m \pi / \lambda), \\
c_{2}=[(M-m) /(M+m)]^{1 / 2} \cos (2 m \pi / \lambda),
\end{gathered}
$$

and

$$
\begin{gathered}
c_{1}=\left[(M+m)^{1 / 2} \cos [(2 m+1) \pi / \lambda]\right. \\
c_{2}=\left[(M+m)^{1 / 2} \cos [(2 m+1) p i / \lambda) .\right.
\end{gathered}
$$

The first set (42) furnishes a finite Hessian matrix, if $M \neq m$; if $m=M$ this matrix has a divergent behavior. The second set (43) generates a null Hessian matrix that cannot be used for the calculation of the integrability coefficient.

For the first case, we have, from (7)

$$
\lambda=2 M /(M-m) .
$$

Therefore, as the Hamiltonian (41) of this system has the degree $k=1$, the non-integrable cases will be those for what

$$
\lambda \neq j(j+1) / 2
$$

$j=0,1,2, \ldots$. Therefore, if

$$
\mu=M / m \neq j(j+1) /[j(j+1)-4]
$$

the system will be a non-integrable one.

Consequently, Theorem I defines the following possible integrability domain for the SAM system: $\mu \in[1,3]$, at the points where $\mu=j(j+1) /[j(j+1)-4]$. For the case where $\mu=3$, we can find the second integral of motion that proves the integrability of the system. It has the form:

$$
I=-\left(r^{3} / 2\right) \theta^{\prime} \operatorname{sen}(\theta / 2)+g r^{2} \operatorname{sen}(\theta / 2) \cos ^{2}(\theta / 2)+r^{2} r^{\prime} \theta^{\prime} \cos (\theta / 2)
$$

and can be found by several methods including the Noether symmetries. The Noether symmetry analysis allows us to say also that there is no additional first integral that is linear in $r^{\prime}$, except for the case $\mu=3$ [19]. This result does not forbid the existence of a second integral of motion with higher order in $r^{\prime}$. However, numerical experiments made by Tufillaro [34], by using the Poincaré sections, suggest that, except for the cases where $\mu=1$ (possibly) and $\mu=3$ (surely), the system is a non-integrable one. 
IV. Homogeneous Hamiltonians with $\mathbf{N}$ degrees of freedom

A recent theorem demonstrated by Yoshida [14] permits the construction of a direct procedure for determining the non-integrability domains of a homogeneous Hamiltonian system with $\mathrm{N}$ degrees of freedom:

\section{Theorem II}

Let $V\left(q_{i}\right)$ be a homogeneous potential of integer degree $k$, with $k \neq 0, \pm 2$, for the following hamiltonian system with $N$ degrees of freedom:

$$
H=p_{i}^{2} / 2+V\left(q_{i}\right) .
$$

We define

$$
\Delta \rho_{i}=\left[1+8 k \lambda_{i} /(k-2)^{2}\right]^{1 / 2}
$$

where $\lambda_{i}$ are the eigenvalues of the Hessian matrix $\mathbf{V}_{i j}\left[q_{i}=c_{i}\right]$, and $c_{i}$ are the solutions of

$$
c_{i}=\left(\partial V / \partial q_{i}\right)\left(c_{i}\right) .
$$

If the $n$ numbers $\left(\left(\Delta \rho_{1}, \ldots, \Delta \rho_{n}\right)\right.$ are rationally independent, then the hamiltonian system (48) has no additional global analytical integral, $I\left(q_{i}, p_{i}\right)=$ constant, besides the Hamiltonian itself.

Some observations can be made about this theorem: i) The quantities $\Delta \rho_{i}=\rho_{i+n}-\rho_{i}$ are the differences between the pairs of the so-called Kowalevskaia exponents $\left(\rho_{1}, \rho_{2}, \ldots, \rho_{2 n}\right)$ for this system. They emerge from the Painlevé's expansion and characterize the branching of the solution in the complex plane $t$. They have, in this case, the pairing property

$$
\rho_{i}+\rho_{i+n}=2 g+1(i=1, \ldots, n)
$$

where $g=2 /(k-2)$;

ii) Due to this pairing property $\rho_{i+n}$ and $\rho_{i}$ can be rational numbers iff the differences $\Delta \rho_{i}$ are rational numbers. One can supposing also that $\rho_{n}$ is rational; it has the form $\Delta \rho_{n}=(3 k-2) /(k-2)$;

iii) If $n=2$, and $k=0, \pm 2$, Theorem II leads to the non-integrability of the system if the nontrivial $\Delta \rho_{i}$ is an irrational or complex number.
For calculating the non-integrability domain of a $n$ degree of freedom homogeneous Hamiltonian system we can use the following algorithm:

Step 1:

Solve and fix a solution $c_{i}$ of the algebraic equations

$$
\partial V\left(c_{i}\right) / \partial c_{i}=c_{i} .
$$

Step 2:

Let $\mathbf{V}_{i j}\left(c_{i}\right)$ be the Hessian matrix of $V\left(q_{i}\right)\left[q_{i}=c_{i}\right]$. Compute the eigenvalues $\left(\lambda_{1}, \ldots, \lambda_{n}\right)$ of this matrix.

\section{Step 3:}

The pair of KE $\left(\rho_{i}, \rho_{i+n}\right)$ are the two roots of the quadratic equation

$$
\rho^{2}-(2 g+1) \rho+g(g+1)\left(1-\lambda_{i}\right)=0,
$$

so that the $\Delta \rho_{i}$ can be calculated by

$$
\rho_{i}=\left[1+8 k \lambda_{i} /(k-2)^{2}\right]^{1 / 2},(i=1, \ldots, n) .
$$

Step 4:

Find the non-integrability domains, where the $n$ numbers $\left(\Delta \rho_{1}, \ldots, \Delta \rho_{n}\right)$ are rationally independent.

Few specific cases of homogeneous hamiltonian systems with three degrees of freedom were studied by using Theorem II. Yoshida [14] showed that the potential

$$
V=q_{1}^{2} q_{2}^{2}+q_{2}^{2} q_{3}^{2}+q_{3}^{2} q_{1}^{2},
$$

leads to

$$
\left\{\lambda_{1}, \lambda_{2}, \lambda_{3}\right\}=\{2,-1,3\}
$$

and to

$$
\left\{\Delta \rho_{1}, \Delta \rho_{2}, \Delta \rho_{3}\right\}=\left\{(17)^{1 / 2},(-7)^{1 / 2}, 5\right\} .
$$

The fact that these quantities are not rationally dependent shows that the potential (53) is a non-integrable one. In the same reference, Yoshida made the conjecture that the potential

$$
V=\left(-q_{1}\right)^{4}+\left(q_{1}-q_{2}\right)^{4}+\ldots+\left(q_{n-1}-q_{n}\right)^{4}+q_{n}^{4},
$$

where $n$ is an odd integer, would be non-integrable for any value of $n$. He arrived at this conclusion by calculating the quantities $\Delta \rho_{i}$ and by examining their rational dependence. 
Ferrándiz and Sansaturio [23] analysed the problem of the motion of a satellite (point mass) around a planet of arbitrary shape, for the specific case where it occurs a perturbation produced only by the term $J_{22}$. They reduced the original potential to the homogeneous form $V=a\left(x^{2}-y^{2}\right) / r^{5}$, where $r=\left(x^{2}+y^{2}+z^{2}\right)^{1 / 2}$. It can be showed that

$$
\left\{\lambda_{1}, \lambda_{2}, \lambda_{3}\right\}=\{7 / 3,-4,5 / 3\} \Longrightarrow\left\{\Delta \rho_{1}, \Delta \rho_{2}, \Delta \rho_{3}\right\}=\left\{(-31)^{1 / 2} / 5,11 / 5,(-15)^{1 / 2} / 5\right\}
$$

and, therefore, by Theorem II, the non-integrability of the system is proved. Let us consider the application of Theorem II to the class of Hamiltonians with the potential

$$
V=q_{1}^{m} q_{2}^{n} q_{3}^{p}
$$

with $k=m+n+p$ and $k \neq 0,2$.

If we follow the steps of the procedure exposed above, we get the following solutions for (50):

$$
\begin{gathered}
c_{1}=\left[p^{-p} n^{-n} m^{p+n-2}\right]^{[1 / 2(k-2)]} \\
c_{2}=\left[p^{-m+n-2} n^{-n} m^{-m}\right]^{[1 / 2(k-2)]} \\
c_{3}=\left[p^{-p} n^{m+p-2} m^{p+n-2}\right]^{[1 / 2(k-2)]}
\end{gathered}
$$

The Hessian matrix $\mathbf{V}_{i j}\left(c_{1}, c_{2}, c_{3}\right)$, which is necessary for applying step 2, will be

$$
\left|\begin{array}{ccc}
n-1 & n^{1 / 2} m^{1 / 2} & n^{1 / 2} p^{1 / 2} \\
n^{1 / 2} m^{1 / 2} & m-1 & m^{1 / 2} p^{1 / 2} \\
n^{1 / 2} p^{1 / 2} & m^{1 / 2} p^{1 / 2} & p-1
\end{array}\right|
$$

The characteristic equation coming from this matrix is

$$
\lambda^{3}+\lambda^{2}(3-k)+\lambda(3-2 k)+(1-k)=0,
$$

and their roots are:

$$
\lambda_{1}=k-1=m+n+p-1, \lambda_{2}=\lambda_{3}=-1 .
$$

Step 3 leads to

$$
\Delta \rho_{1}=\frac{(3 k-2)}{(k-2)}, \Delta \rho_{2}=\Delta \rho_{3}=\left[k^{2}-12 k+4\right]^{1 / 2} /(k-2)
$$

According the last step, if the ratio between $\Delta \rho_{1}$ e $\Delta \rho_{2}$ is non-rational the system will be non-integrable. This situation will occur in most cases. Consider now the particular case where $m=n=p=1, k=3$, i.e, with the potential

$$
V=q_{1} q_{2} q_{3}
$$

From (60), we get

$$
\Delta \rho_{1}=7, \Delta \rho_{2}=\Delta \rho_{3}=(-23)^{1 / 2}
$$

and the system is a non-integrable one. There are cases that are not in the non-integrability domain, for example if $k=-3$ or $k=12$. In these cases, by using Theorem II, we can say nothing about the integrability of the potentials.

\section{Conclusion}

The Ziglin-Yoshida method for the analysis of the non-integrability domains of hamiltonian systems has the advantage, in comparison with Melnikov's method, for example, that it can be applied to more general situation, not only to perturbed Hamiltonians. In contrast with the singularity analysis, it is a global procedure that permits the precise definition of non-integrability regions. Theorems I and II are direct algorithmic procedures for analyzing the integrability of homogeneous hamiltonian systems.

In the most general formulation of Ziglin the theorem has not a simple way of application; the method involves also, in many situations, the resolution of nontrivial algebraic equations. Except for the case of homogeneous Hamiltonians, where Yoshida's theorems can 
be applied, few systems were already studied. We also note that the integrable cases are not fully determined by this method; only domains (in parameter space), where the integrable systems could appear, can be established. Furthermore, the identification of first integrals, a necessary step for the final proof of integrability, has to be made by using other methods. But the Ziglin-Yoshida analysis is without doubt a powerful and interesting global method, based on the linear analysis of the variational equations, for analyzing the non-integrability of Hamiltonian systems. The mathematical meaning of this method and the physical aplications will be possibly extended in the near future.

We are grateful to Haruo Yoshida for introducing us to this method.

\section{References}

1 T. Bountis, B. Grammaticos and A. Ramani, Phys. Rep. 180, 159 (1989).

2 J. Hietarinta, Phys. Rep. 147, 87 (1987).

3 . I. C. Moreira, Had. Journal 7, 475 (1985).

4 . J. M. Strelcyn and S. Wojciechowski, Phys. Lett. A133, 207 (1988).

5 . M. A. Almeida, I. C. Moreira and M. E. Magalhães, J. Math. Phys. 36, 1854 (1995).

6 I. C. Moreira, Rev. Bras. Fís. 21, 60 (1991).

7 P. D. Lax, Commun. Pure Appl. Math. 21, 467 (1968).

8 T. Carlemann, Acta Mathematica 59, 63 (1932).

9 L. Brenig, Phys. Lett. A133, 378 (1988).

10 V. Arnold, Les methodes mathematiques de la Mécanique Classique, Editions Mir, Moscou, 1976.

11 J. Guckenheimer and P. Holmes, Nonlinear oscillations, dynamical systems and bifurcations of vector fields, Springer-Verlag, New York, 1983.
12 S. L. Ziglin, Func. Anal. Appl. 16, 181 (1983); 17, 6 (1983).

13 H. Yoshida, Physica D29, 128 (1987).

14 H. Yoshida, Phys. Lett. A141, 108 (1989).

15 H. Ito, Kodai Math. J. 8, 120 (1985).

16 B. Grammaticos, J. Hietarinta, A. Ramani and H. Yoshida, Physica A144, 310 (1987).

17 H. Yoshida, Phys. Lett. A120, 388 (1987).

18 J. Casasayas, A. Nunes and N. Tufillaro, J. Physique 51, 1693 (1990).

19 M. A. Almeida and I. C. Moreira, J. Physique I 1, 711 (1991).

20 H. Yoshida, Commun. Math. Phys. 116, 529 (1988).

21 M. A. Almeida, I. C. Moreira and H. Yoshida, J. Phys. A: Math. Gen. 25, L227 (1992).

22 D. Roekaerts and H. Yoshida, J. Phys. A: Math. Gen. 21, 3547 (1988).

23 J. M. Ferrandiz and M. E. Sansaturio, Phys. Lett. A207, 180 (1995).

24 B. Grammaticos, A. Ramani and H. Yoshida, Acta Appl. Math. 8, 75 (1987).

25 C. Stormer, Arch. Sci. Phys. Nat. 24, 113 (1907).

26 A. N. Krylov, Phys. Uspekhi 36, 226 (1993).

27 L. Jimenez-Lara and E. Pina, Celestial Mech. 49, 327 (1990).

28 G. Contopoulos and L. Vlahos, J. Math. Phys. 16, 1469 (1975).

29 A. J. Dragt and J. M. Finn, J. Geophys. Res. 81, 2327 (1976).

30 C. Jung and H. Scholz, J. Phys. A: Math. Gen. A21, 2301 (1988).

31 C. Jung and B. Rückerl, J. Phys. A: Math. Gen. 27, 6741 (1994)

32 M. Noguera, Doctoral thesis, University of Barcelona, 1986.

33 S. G. Matinyan, E. B. Prokhorenko and G. K. Savvidy, Nucl. Phys. B298, 414 (1988).

34 N. Tufillaro, J. Physique 46, 1495 (1985). 